Accelerator Department

BROOKHAVEN NATIONAL LABORATORY

Associated Universities, Inc. Upton, L.I., N.Y.

E $P \& S$ DIVISION TECHNICAL NOTE

No. 25

G. Danby, J. Jackson and E.C. Raka

February 25, 1969

\title{
AGS LATTICE CORRECTIONS AND TUNING
}

The results of the excitation of the new backleg winding system verify the value of this method of increasing the intensity of the AGS, but of even more interest are the important implications regarding further increases and a really quantitative study of high intensity effects.

This brief descriptive note will explain the ideas involved. It will be followed shortly by a detailed report.

The lattice design of the AGS is based on the dynamic magnetic field characteristics. However, at low dynamic field values (near injection) the eddy current and remanent fields have a major perturbing effect on synchrotron properties, just when the beam is most vulnerable. Eddy currents in the vacuum chambers and end plates depress $k$ values and also introduce some higher multipole nonlinearity. The $v$ values of the machine are affected by eddy currents, but the lattice symmetries are substantially maintained. Remanence, on the other hand, also introduces effects which depend on whether the hyperbolic poles open or close with respect to the backleg magnet yokes. The open or closed configuration does not vary in step with the cell structure. The resulting $\beta$ distortion is equivalent to a substantial reduction in the available acceptance. In addition to $\beta_{\max }$ being made larger $\beta_{\min }$ is of course made sma1ler.

The AGS has always had one pair of tuning quadrupoles per superperiod. In the course of tuning up the machine these quadrupoles themselves produce large $\beta$ distortions. A considerably greater number is necessary to give high enough multiplicity so that tuning itself is not a major source of distortion.

In summation, the two problems of $\beta$ distortion and tuning of the rapidly changing $v$ values near injection lead to the formidable requirement of introducing very large numbers of $\beta$-correction and tuning quadrupoles into the existing machine. Indeed such magnets have been planned as part of the conversion. 
The idea proposed by one of the authors (G. Danby) was simply that the excitation of the 240 main magnets could be varied to give the desired focusing strengths while the resulting dipole perturbations could be easily handled. With combined function magnets any variation in gradients leads, of course, to a proportional variation in the dipole fields. Although AG machines are very sensitive to dipole field perturbations, in this case the orbit distortion is quite small. This comes about because one is varying the fields with very high multiplicity compared to the $v$ values of the machine (i.e. $N=60$, which is the number of cells for the AGS, versus $v<9$ ) and as a result the forcing function has this relatively high frequency.

A study of such systems was carried out by G. Danby and J. Jackson and extensive computations were made using the SYNCH Program and Fourier analysis. The results were the following:

(1) 12 ampere turns in a circuit around a11 the backlegs equalize the gradients to completely remove the backleg $\beta$ distortion. Because of the structure of the lattice, this in turn leads to some equilibrium orbit distortion. Therefore, with the gradients corrected, the orbit distortion was Fourieranalyzed and showed amplitudes of $+0.6 \mathrm{~cm}$ for $12 \theta,-0.14 \mathrm{~cm}$ for $24 \theta$, and $-0.04 \mathrm{~cm}$ for 360. Imposing a sin $12 \theta$ correction of 5 ampere turns around 24 equally spaced magnets reduces the maximum orbit displacement below 100 mils. This modest residue is predominantly $24 \theta$ and in fact could be removed if desired. Also the $\beta$ distortion with the $12 \theta$ orbit correction imposed is only about $1 \%$.

(2) Other windings which are constructed to alternate with the ce11 structure can be used for $v$ tuning. Since this has the full $N=60$ multiplicity of the machine, there is negligible orbit or $\beta$ distortion. This arrangement can be used for an extended class of $v$ variations and the circuit is completely non-inductively coupled to the primary magnet current. This makes precise programming of a power supply for dynamic tuning very easy. For a large $\Delta v$ of 0.3 , the equilibrium orbit is only distorted by $\sim 20$ mils.

(3) In addition to these focusing and distortion corrections, Fourier analysis was used with a particularly simple approach to harmonic orbit corrections.

The phase-amplitude solutions elegantly and accurately describe synchrotron behavior. The azimuthal modulation of the phase and of the amplitude coordinates can be incorporated into a new set of coordinates variable in real space so that the behavior in the new coordinate system can be described by harmonic functions rather than being governed by Hil1's Equation. 
In the present work the azimuthal modulation was ignored for computational purposes. This modulation is at a frequency $(\mathrm{N}=60)$ five to ten times higher than the harmonics one is interested in correcting. Any systematic computational error will essentially apply to an orbit distortion and to its compensating $180^{\circ}$ phase shifted correction. It should be stressed that the approximation is only in the computational stage. Having found the appropriate orbit corrections in this way, the actual corrected orbit properties are calculated using the SYNCH program. It was decided to proceed and design the windings described and arrange the yoke winding terminals in a manner to permit many future horizontal harmonic perturbations to be easily installed.

The work described was officially reviewed last year and the decision to proceed with the installation was made. The actual work in the ring itself was completed after the September 1968 shutdown. To date the only testing was to check the correctness of the layout and installation by looking at induced voltages. No errors were found! Testing is now beginning and we hope shortly to dynamica11y excite the tuning windings.

The main problem turned out to be that of finding a power supply to feed the $\beta$ correction windings which have a back EMF of $\approx 20$ volts at injection and $\approx 100$ volts at maximum $\dot{B}$. There was nothing available that would track this variation and furnish six amps into $41 \Omega$ so a temporary compromise was made. Two 250 volt supplies were connected in series with $32 \Omega$ of resistance to the $\beta$ windings. When running at a total of $\approx 470$ volts $6^{+}$amperes through two turns are obtained at injection with a drop of $\approx 18 \%$ at maximum $\dot{\mathrm{B}}$.

The $\beta$ correction winding was switched on as per design and the $v$ tuning winding has been dc excited. The early losses were greatly reduced, corresponding to a $25 \%$ to $30 \%$ increase in beam held.

The fact that these windings have not been studied but were only essentially switched on as designed and installed speaks very highly for the quality of the work of the AGS staff involved. Significantly, those present observed a very stable operation and every indication is that the potential for further significant intensity gains is very good. Even at intensities of $3 \times 10^{12}$ a factor of two reduction in brightness gave a factor of two less beam with no retuning of the machine. Although this result must be somewhat qualified in detail, its implications for the future are clearly very great.

Distr: Accelerator Dept. Staff 